\title{
Use of triiodothyronine to treat critically ill COVID-19 patients: a new clinical trial
}

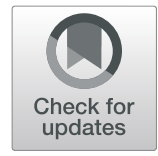

\author{
Constantinos Pantos (D), loulia Tseti and Iordanis Mourouzis
}

\section{Letter}

As COVID-19 disease spreads, the number of critically ill patients requiring intensive care unit (ICU) support increases and mortality is remarkably high, calling for novel effective therapies to treat sepsis and septic shock in COVID-19 patients.

From the pathophysiologist's view, sepsis in viral infected patients results in cell damage due to both uncontrolled viral entry/replication and hypoxia. Hypoxia and viral infectivity share common pro-apoptotic signaling pathways, such as p38 mitogen-activated protein kinase (p38 MAPK) [1, 2] Fig. 1. In addition, inotropes and vasoactive agents, often used in ICU treatments, can increase tissue injury and viral load via 338 MAPK activation $[1,2]$. Therefore, a vicious cycle is established in viral sepsis leading to high mortality despite the current therapies. Epinephrine and dobutamine use in sepsis is reported to be associated with increased mortality and atrial fibrillation [3].

Under acute illness, such as sepsis, myocardial infarction, and trauma, deregulation of thyroid hormone (TH) metabolism (non-thyroidal illness syndrome, NTIS) occurs and low circulating triiodothyronine (T3) is associated with increased mortality
[4]. NTIS is of physiological relevance in cell defense, and it is now recognized that $\mathrm{TH}$ can increase tolerance of the cell to hypoxia via suppression of p38 MAPK activation and promote tissue repair through controlled Akt activation [1]. This novel reparative action of $\mathrm{TH}$ is currently investigated in the ThyRepair trial (EudraCT: 2016-00063140) in patients with anterior STEMI undergoing angioplasty. In this study (almost completed), no major adverse effects have been observed.

TH also appears to prevent herpes simplex virus infectivity and potentiate host defense by increasing natural killer cells $(\mathrm{NK})$ and enhancing the stimulatory effect of interferon on NK cells [5]. On the contrary, corticosteroids which are often used in ICU therapies suppress lung inflammation but also inhibit immune response and pathogen clearance and their use remains questionable.

On the basis of this evidence, a new phase II randomized, double blind, placebo controlled trial (Thy-Support, ClinicalTrials.gov Identifier: NCT04348513) is going to investigate the effect of intravenous high dose T3 (UniPharma S.A., Greece) for enhancing recovery of critically ill COVID-19 patients.

\footnotetext{
* Correspondence: cpantos@med.uoa.gr

Department of Pharmacology, Medical School, National and Kapodistrian

University of Athens, 75 Mikras Asias Ave.,11527 Goudi, Athens, Greece
} 


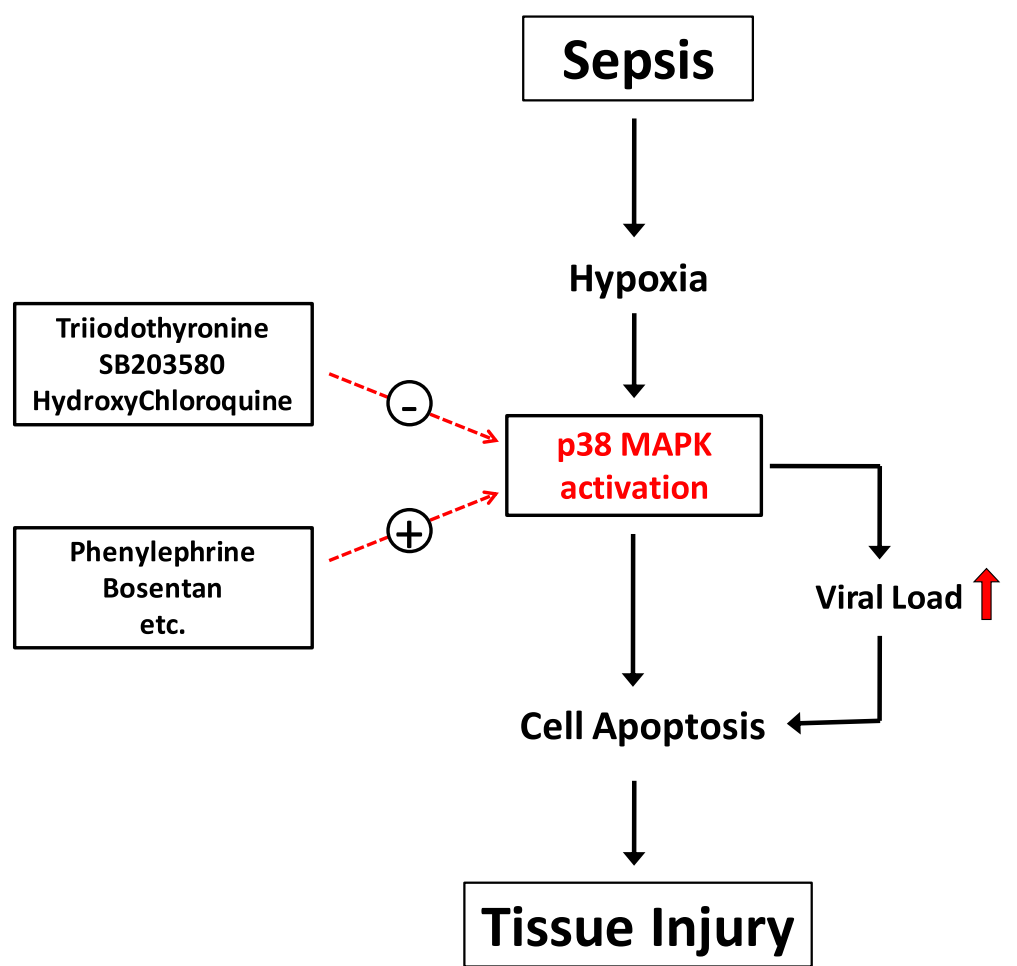

Fig. 1 Proposed scheme showing that hypoxia and viral infectivity share common pro-apoptotic signaling pathways, such as p38 MAPK

\section{Acknowledgements}

Not applicable

\section{Authors' contributions}

$\mathrm{CP}$ and IM have made substantial contributions to the conception and writing of the work. IT was a contributor in reviewing and writing the manuscript. All authors read and approved the final manuscript.

\section{Funding}

Not applicable

Availability of data and materials

Not applicable

Ethics approval and consent to participate

Not applicable

\section{Consent for publication}

Not applicable

\section{Competing interests}

The following pending patents are relevant to the work in this manuscript: - PCT/EP2019/087056. L-triiodothyronine (T3) for use in limiting microvascular obstruction

- Greek Patent Office, number of case: 22-0002577373. Composition comprising L-triiodothyronine (T3) for use in the treatment of critically ill patients with coronavirus infection.

$I T$, managing director of Uni-Pharma, is the sponsor and owner of these patents. CP and IM are the inventors and hold royalties in relation to these patents.
Received: 22 April 2020 Accepted: 30 April 2020

Published online: 08 May 2020

References

1. Pantos C, Mourouzis I. Translating thyroid hormone effects into clinical practice: the relevance of thyroid hormone receptor a1 in cardiac repair Heart Fail Rev. 2015;20(3):273-82.

2. Marchant D, Dou Y, Luo H, Garmaroudi FS, McDonough JE, Si X, et al. Bosentan enhances viral load via endothelin-1 receptor type-A-mediated p38 mitogen-activated protein kinase activation while improving cardiac function during coxsackievirus-induced myocarditis. Circ Res. 2009;104:81321.

3. Sato R, Ariyoshi N, Hasegawa D, Crossey E, Hamahata N, Ishihara T, et al. Effects of inotropes on the mortality in patients with septic shock. J Intensive Care Med 2019:885066619892218. doi: https://doi.org/10.1177/ 0885066619892218.

4. Padhi R, Kabi S, Panda BN, Jagati S. Prognostic significance of nonthyroidal illness syndrome in critically ill adult patients with sepsis. Int J Crit IIIn Inj Sci. 2018:8:165-72.

5. Varedi M, Moattari A, Amirghofran Z, Karamizadeh Z, Feizi H. Effects of hypo- and hyperthyroid states on herpes simplex virus infectivity in the rat. Endocr Res. 2014;39:50-5.

\section{Publisher's Note}

Springer Nature remains neutral with regard to jurisdictional claims in published maps and institutional affiliations. 